\title{
Nanostructured Organic Alkali-Soluble Silicates for
}

\section{Industrial Application}

\author{
Pavel Kudryavtsev and Oleg Figovsky \\ Polymate Ltd., Israel Research Center, Migdal Haemek 23100, Israel
}

\begin{abstract}
The work describes the properties of soluble organic silicates and their applications to obtain nanocomposite materials. We analyzed the properties of the water-soluble high-modulus silicate systems and their technology for producing. The aim of this paper is the comparison properties of binders based on liquid glass containing strong organic bases silicates. We have shown how these systems are transformed from lower to higher oligomers through the formation of the silica sol and the implementation of the sol-gel process for these oligomers. We have conducted advanced research of various aspects of the use of these materials as the binder. Advantages of strong organic bases silicates in the preparation of heat resistant, nanocomposite materials are shown. Ways to obtaining quaternary ammonium silicates and their use to produce nanocomposites are proposed. Products obtained in this way can be used as a binder in the preparation of nanostructured composite materials, water-based paints, coatings, etc. Modifiers have been proposed for making of hybrid nanostructured composite materials by a sol-gel process. There have been shown of structuring phenomena some aspects, synthesis and application of hybrid materials based on silica with grafted polymers. It has been shown, the possibility of modifying compositions using the nanostructuring agents such as tetrafurfuryloxysilane. This paper also describes methods for the synthesis of products for modifying a sol-gel process using organic soluble silicates. We are displaying their use for the production of new nanocomposite materials and coatings for protection against various external factors.
\end{abstract}

Key words: Organic alkali silicates, corrosion-resistant materials, nano structuring materials, coating.

\section{Introduction}

Despite the development of new types of corrosion-resistant materials are special types of steel, polymer composites, polymer cements, glass-reinforced plastics, etc., materials based on liquid glass not lost their significance [1-3]. This is due to the fact that effective new polymeric materials, road deficient require much labor in many cases do not have the necessary resource base to have enhanced toxicity. Acid-resistant, prepared with liquid glass, devoid of these shortcomings.

The main types of acid-resistant materials are acid-resistant cements, putties, concretes and coatings. Acid and chemical resistant coatings are divided into two main groups-polymer concrete made with organic synthetic resins (furan, polyester, urea, etc.) and polysilicate composite coatings based on sodium

Corresponding author: Kudryavtsev Pavel, doctor of science, professor, research fields: nanotechnology, physical chemistry, sol-gel processes. or potassium liquid glass.

Polysilicate composite coatings are prepared from acid-resistant placeholders of different granulometric composition: coarse aggregate-crushed granite, fine-quartz sand fine ground placeholder curing initiator-sodium hexafluorosilicate and special additives of plasticizers, sealants, water repellents, etc. [2]. In industry the greatest distribution has an acid-resistant quartz cement hexafluorosilicate. It is a mixture consisting of fine silica sand and sodium hexafluorosilicate. The mixture shuts with potassium or sodium liquid glass, after which hardening the air forms an acid-stone. The content of sodium hexafluorosilicate, in cement is $4 \mathrm{wt} \%$ (material of 1 type for acid) or $8 \mathrm{wt} \%$ (material of 2 types for acid and solutions). Acid resistant quartz materials, based on sodium hexafluorosilicate, are used as a binder for laying the lining of chemically resistant materials (bricks, tiles), in the protection of buildings, chemical equipment and also for the production of acid-proof 
coatings.

The most difficult task is the protection of chemical equipment operating in an environment of $70 \%$ sulfuric acid at its boiling point. Such processes are widely used in fine chemical, such as decarboxylation processes of organic acids, ester saponification process, the depolymerization of polyesters, etc. To protect such apparatus we have developed a composition comprising from fine zircon $\mathrm{ZrSiO}_{4}$ (88\%), sodium hexafluorosilicate (2\%) and liquid glass $(10 \%)$. Such a composition is sustained, in real conditions, up to 50 cycles in a medium of $70 \%$ sulfuric acid, with a cycle time of $10 \div 12$ hours.

In recent years it has begun to develop application of silicate polymer composite materials which are water soluble silicates with additives of active substances furan series. They operate under acidic and neutral media and under the influence of elevated temperatures. The materials are cheap and easy to manufacture non-toxic, non-flammable. Cost of polymer silicate materials is commensurate with the cost of cement concrete and several times lower than the cost of polymer concrete. Silicate polymer materials as concrete, mortar, putties were using for making constructions of various purpose, monolithic and tile lining. There is a certain perspective, in composite materials based on liquid glass binder modified with FA (furfuryl alcohol).

A significant increase in strength, thermal and fire resistance, of silicate matrix is achieved by introducing into the composition of esters of orthosilicic acid (tetrafurfuryloxysilanes-TPS) and furfuryl alcohol. Effect is achieved by strengthening of contacts between the globules of silica gel and modification of the alkaline component, due to the "inoculation" of the furan radical. Introduction to the binder, additives TFS leads to the formation of nanoparticles of $\mathrm{SiO}_{2}$, and $\mathrm{FA}$, which fills the matrix, and forms the crosslinked polymer. These particles act as centers of nucleation and crystallization. Adding TFS increases the mechanical and chemical resistance of the binder, and this approach began to be widely used for the preparation of acid-resistant coatings [4, 5].

For increasing the strength, the acid resistance, heat resistance and flame resistance of construction materials and structures of them, TFS are input to the binder composition. They are synthesized by transesterification of tetraethoxysilane and FA.

Composition of the resulting binder comprises: a liquid glass - $80 \div 95 \mathrm{wt} \%$ TFS - $2 \div 7 \mathrm{wt} \%$, hardener, sodium hexafluorosilicate $-13 \mathrm{wt} \%$. Thus as part of the liquid glass used organic alkali liquid glass, wherein the organic cation is taken 1,4-diazabicyclo[2.2.2] octane-1,4-diium or 1,5-diazabicyclo[3.3.3] undecane-1,5-diiumsilicates $2 \div 4$ wt $\%$ (Fig. 1).

Water-soluble silicate containing an organic alkali cation was prepared by reacting the salt of an organic quaternary ammonium derivative with an amorphous silica. Soluble organic alkali silicates, such as TBAS (tetrabutylammonium silicate) were used as a binder component for self-extinguishing $[6,7]$.

Developed nanostructuring binder prepared laminar mixing liquid glass containing cations of alkali metals such as sodium; TFS (tetrafurfurylsilicate) and a water-soluble silicate, a water-soluble silicate containing an alkali organic cation such as diazabicyclo[2.2.2] octane-1,4-diium or 1,5-diazabicyclo[3.3.3] undecane-1,5-diium [8].

After mixing all components of the binder, it is necessary to use for $2 \div 3$ hours. Adding of hardener is carried out together with fine ground mineral filler.

Introduction of tetrafurfurylsilane which is nanostructured component in the binder leads to the formation of nanoparticles of $\mathrm{SiO}_{2}$ and furfuryl alcohol. $\mathrm{SiO}_{2}$ nanoparticles act as centers of nucleation and crystallization. Furfuryl alcohol which fills the silica matrix is destroyed, and thus is polymerized. Adding TFS increases the mechanical and chemical resistance of the binder and is widely used for the preparation of acid-resistant coatings. Liquid 

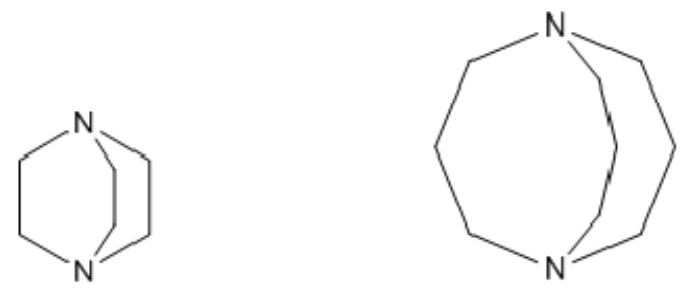

1,4-diazabicyclo[2.2.2] octane 1,5-diazabicyclo[3.3.3]undecane
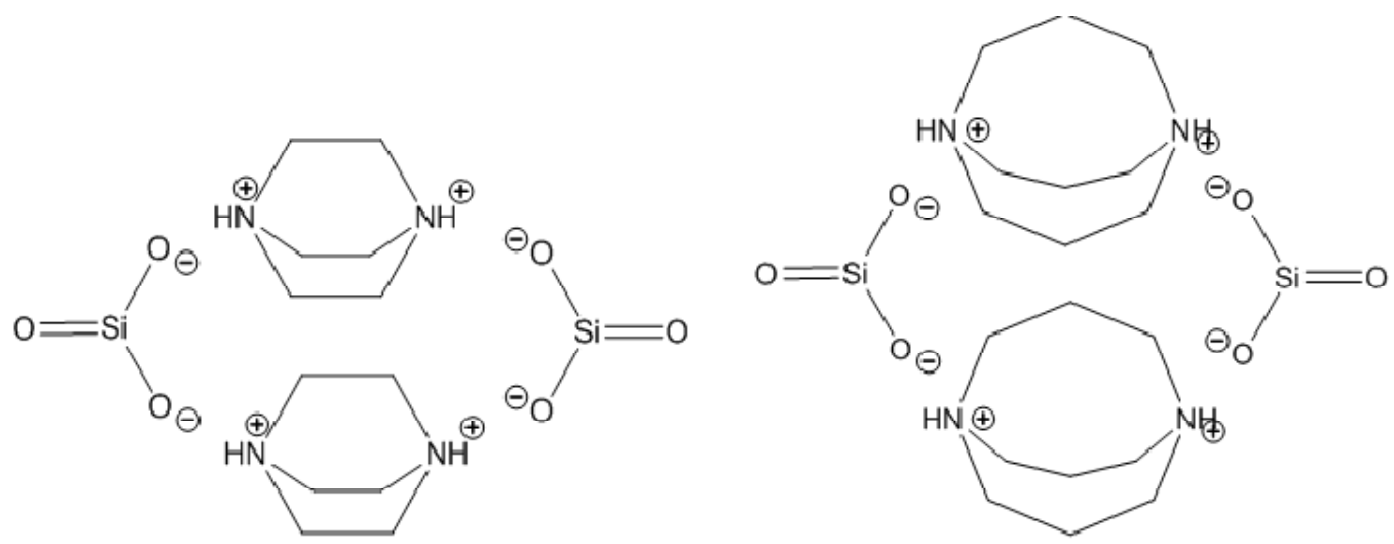

1,4-diazabicyclo[2.2.2] octane-1,4-diium silicate 1,5-diazabicyclo[3.3.3] undecane-1,5-diium silicate

Fig. 1 1,4-diazabicyclo[2.2.2]octane and 1,5-diazabicyclo[3.3.3] undecane. 1,4-diazabicyclo[2.2.2]octane-1,4-diiumsilicate and 1,5-diazabicyclo[3.3.3] undecane-1,5-diiumsilicate.

glass with an additive of 1,4-diazabicyclo[2.2.2] octane-1,4-diium silicate is compatible with an aqueous dispersion of chloroprene rubber and polyurethanes, as well as most synthetic latexes based rubbers. Such composite materials based on inorganic and organic interpenetrating polymer networks are described in Refs. [9, 10].

\section{Synthesis of Strong Organic Bases Silicates}

An important aspect of the successful application of organic based silicates is to develop simple and effective method of their synthesis based on available and quite cheap starting materials. We have developed a new method for the synthesis of such compounds. This method consisted of using silica sol, as a raw material in the synthesis of organic silicates.

In interaction of silica sols with strong organic bases, stabilization of sol can only be achieved by recharging its surface. For this purpose, it is sufficient to bring the $\mathrm{pH}$ up to 9 . In these conditions it is possible to obtain stable silicate systems with high silica modulus of $\mathrm{SiO}_{2}: \mathrm{M}_{2} \mathrm{O}$. In this case, his magnitude of the resulting silicate module depends only on the diameter of the sol particles. This relationship is shown in Fig. 2. In fact, Fig. 2 is a state diagram of silicate systems with strong organic bases. The field of this diagram, which lies between the applied line and axis $d(\mathrm{~nm})$, corresponds to the region where stable colloidal polymeric and monomeric silicate systems may exist. Above this line, the corresponding silicate systems are subject to fairly rapid coagulation and gelation. Thus, for silica sol with the recommended limit particle size $d$ of 7 to 8 $\mathrm{nm}$, the limit for the theoretically possible silicate module $\mathrm{SiO}_{2}: \mathrm{M}_{2} \mathrm{O}$ will be in the range of 17 to 19 .

As is known, soluble alkaline silicate systems are extensive and are classified according to the following criteria [11]: 


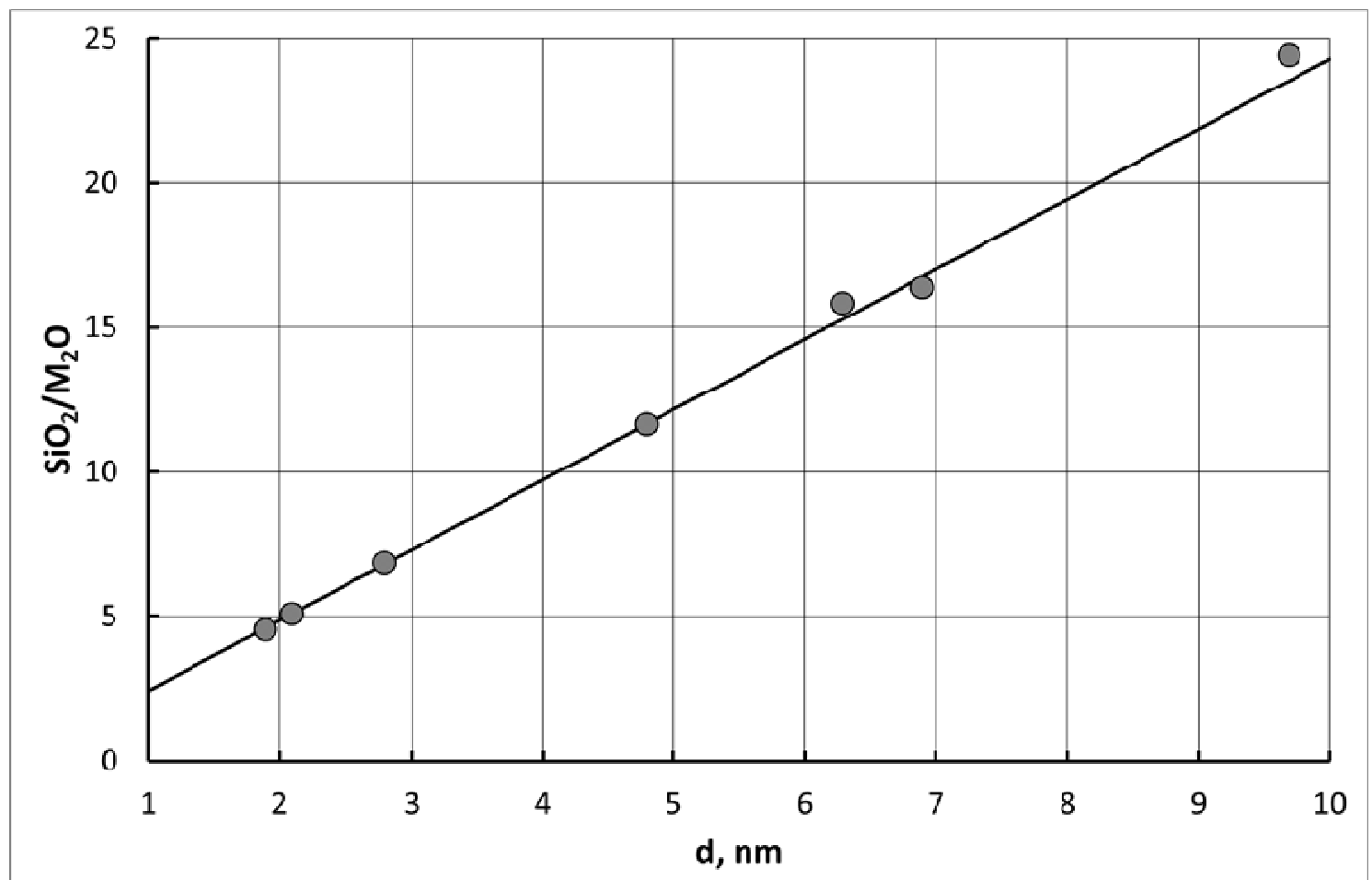

Fig. 2 A graph that shows limit values of $\mathrm{SiO}_{2}: \mathrm{M}_{2} \mathrm{O}$ silica modulus which can be obtained using silica sol neutralized by an organic base to $\mathrm{pH} 9$.

(a) by degree of polymerization $(l)$ of silica, i.e., by an average number of silicon atoms that form during polymerization a continuous system of siloxane bonds $\equiv \mathrm{Si}-\mathrm{O}-\mathrm{Si} \equiv$; polymerization of silica is accompanied by increase in its molecular weight (M), and at high degrees of polymerization, by increase in the size $(d)$ of colloidal silica particles; at a certain degree of polymerization $(l)$, colloidal silica appears in alkali silicate systems as a sol and as a highly hydrated silica:

$$
\begin{aligned}
& \text { Monomers } \rightarrow \text { Lower Oligomers } \rightarrow \text { Higher Oligomers } \rightarrow \text { Colloidalsilica, sols } \\
& (l=1) \quad \rightarrow \quad(l=1 \div 25) \quad \rightarrow \quad\left(\text { polysilicicacids, } M<10^{5}\right) \quad \rightarrow \quad\left(M>10^{5} \text { or, } d>2 \mathrm{~nm}\right)
\end{aligned}
$$

(b) by chemical composition in the course of alkalinity increase, characterized by a molar ratio of $\mathrm{SiO}_{2} / \mathrm{M}_{2} \mathrm{O}$ (silicate module $\mathrm{n}$ ); in the case of

$$
\begin{aligned}
& \begin{array}{l}
\text { Highly alkaline systems } \\
(n<2)
\end{array} \quad \rightarrow \quad \begin{array}{l}
\text { Liquid glasses } \\
(n=2 \div 4)
\end{array}
\end{aligned}
$$

Thus, by analogy with silicate liquid glasses, the optimal values of silica modulus $\mathrm{SiO}_{2}: \mathrm{M}_{2} \mathrm{O}$ for silicates of strong organic bases are in the range of 2 to 4. For certain special cases it can be reduced to $\mathrm{SiO}_{2}: \mathrm{M}_{2} \mathrm{O}$ in the range of 17 to 19 and even higher values. However, such systems are difficult to obtain and they may be sufficiently unstable. The silica modulus $\mathrm{SiO}_{2}: \mathrm{M}_{2} \mathrm{O}$ cannot have a value less than 2 . alkali silicate systems, such systems form a row corresponding to the above four forms of silica:

$$
\rightarrow \begin{aligned}
& \text { Polysilicates } \\
& (n=4 \div 25)
\end{aligned} \quad \rightarrow \quad \begin{aligned}
& \text { Sols } \\
& (n>25)
\end{aligned}
$$

Otherwise conventional molecular silicates which do not possess binding properties are formed.

In this process it is possible to use any type of strong organic bases. Since silica dissolves completely at $\mathrm{pH} 10.7$ to 11.0 and even at high $\mathrm{pH}$ values, it is possible to prepare silicates of organic bases having dissociation constants $\mathrm{pK}_{\mathrm{b}}$ less than 3 . Obtained in this manner compounds will also have a predetermined 
amount of silica modulus expressed by analogy with the silicate modulus of alkali metals in the form of $\mathrm{SiO}_{2}:\left(\mathrm{NR}_{4}\right)_{2} \mathrm{O}$.

\section{Tetrafurfuryloxysilane as Nano Structuring Agent}

With the introduction of specific organic silicate additives such as TFS, it is possible to achieve a significant increase in density and strength of the silicate matrix under stringent conditions. This effect is due to hardening of contact between silicate globules of gel binder and an alkali component because of the "inoculation" of the furan radical [6].

In the hydrolysis process, TPS, is formed active nanoparticles of hydrated silica $\mathrm{SiO}_{2}$ and FA, resulting from this process are created oligomeric nano-films on the surface of the silicate matrix grains. TFS is a kind of micro-crystallizing nucleator which blocks pinholes in the silicate matrix and reduces shrinkage deformation of coating.

In recent years, we began to develop the application of polymer-silicate composite materials, which are water-soluble silicates with active additives of furan series substances. They are operated under acidic and neutral media and exposed to high temperatures. These materials are cheap and easy to produce, non-toxic, non-flammable. The polymer-silicate materials in the form of mortar, are used for the manufacture of various structures, monolithic, and piece lining. There are some prospects for the use of composite materials based on binders of liquid glass, modified by furfuryl alcohol.

A significant increase in strength, heat and fire resistance silicate matrix is achieved by introducing into the composition tetra-furfuryl esters of orthosilicic acid-TFS (Fig. 3). This effect is achieved by strengthening the contacts between the globules of silica and modification of the alkaline component by the emergence of the furan radical. Introduction of TFS additives in the binder leads to the formation of $\mathrm{SiO}_{2}$ nanoparticles and FA. FA fills the pores of the matrix, and forms a crosslinked polymer. Adding TFS increases the mechanical and chemical resistance of the binder, and this approach began to be widely used for the preparation of acid-resistant coatings [12].

This effect can be explained by the following considerations. About the thermal stability of the oxo compounds can be judged by the relative strength of the interatomic bonds $\mathrm{M}-\mathrm{O}$ and $\mathrm{C}-\mathrm{O}$ in their crystal-structure. The bond lengths of $\mathrm{M}-\mathrm{O}$ and $\mathrm{C}-\mathrm{O}$ within the coordination polyhedron can vary considerably, which indicates their power disparities. During the dehydration and heat exposure denticity, a certain part of the ligands may vary. The emerging structure they can start at the same time performs the function of the ligand, and the absence of a solvate. Increasing denticity of the ligands leads to distortion of the oxygen environment of the matrix element or filler, with a corresponding change in the distance of $\mathrm{M}-\mathrm{O}$ and $\mathrm{C}-\mathrm{O}$ in the structure and hence a change in their strength.

To increase the strength, acid-, heat and fire resistance of building materials and designs of them are entered into the binder tetrafurfuryl esters of orthosilicic acid (tetrafurfurylhydroxysilanes-TFS). They are synthesized by transesterification of tetraethoxysilane with FA.

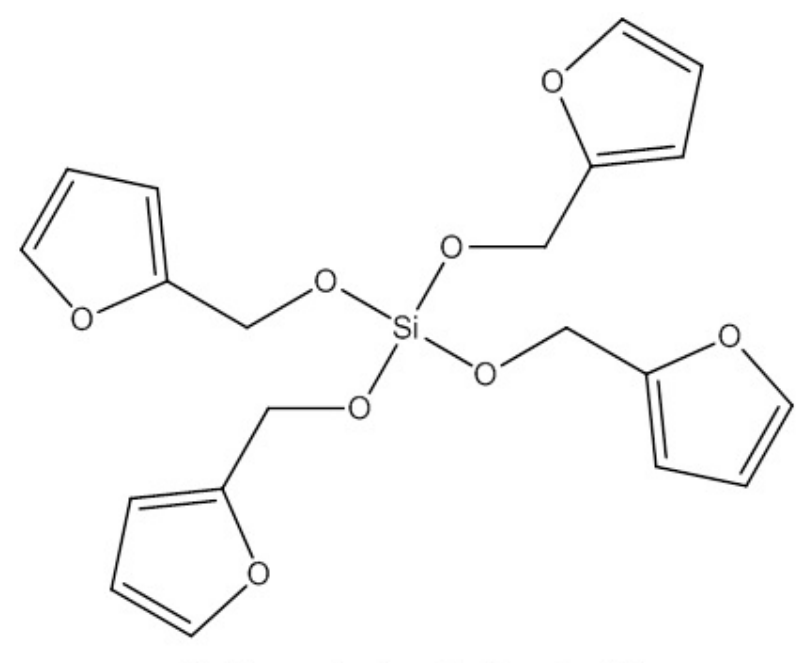

tetrakis(furan-2-ylmethyl) orthosilicate

Fig. 3 Chemical structure of tetrafurfuryloxysilane (tetrakis (furan-2-yl-methyl) orthosilicate). 
The resulting binder comprises: water glass $-80 \div$ 95 wt. $\%$ tetrafurfuryloxysilane (TFS) - $2 \div 7$ wt. $\%$, Curing agent, sodium hexafluorosilicate - 13 wt.\%. Thus as part of waterglass used organic alkali waterglass, wherein the organic cation is taken 1,4-diazabicyclo[2.2.2]octane-1,4-diiumsilicate and 1,5-diazabicyclo[3.3.3] undecane-1,5-diiumsilicate - 2 $\div 4$ wt.\%. Sodium silicate with a cation of 1,4-diazabicyclo[2.2.2] octane-1,4-diium, compatible with aqueous dispersions of polyurethane and chloroprene as well as with the majority of latexes based on synthetic rubbers.

To control the properties of the resulting polysilicate composite coatings and preparation of nanostructured materials with optimal properties requires detailed understanding of the mechanisms of processes occurring in their preparation. This requires details knowledge of the structure of materials used and in particular tetrafurfuryloxysilane. For this were conducted quantum chemical calculations of the structure, the topology and properties of tetrafurfuryloxysilane. The calculations were performed by the method described in Ref. [13]. The calculated molecular structure of tetrafurfuryloxysilane is represented in Fig. 4.

The structure of the molecule is calculated by minimizing the total energy of the molecule by minimizing the gradient and reaches the minimum value. With the use of two variants of the basis of calculation: the original structure, and after application of the procedure of molecular dynamics. Procedure of molecular dynamics included 10,000 iterations, thus these results more accurately describe the topology and energy molecules of tetrafurfuryloxysilane. The results of energy calculations are presented in Table 1.

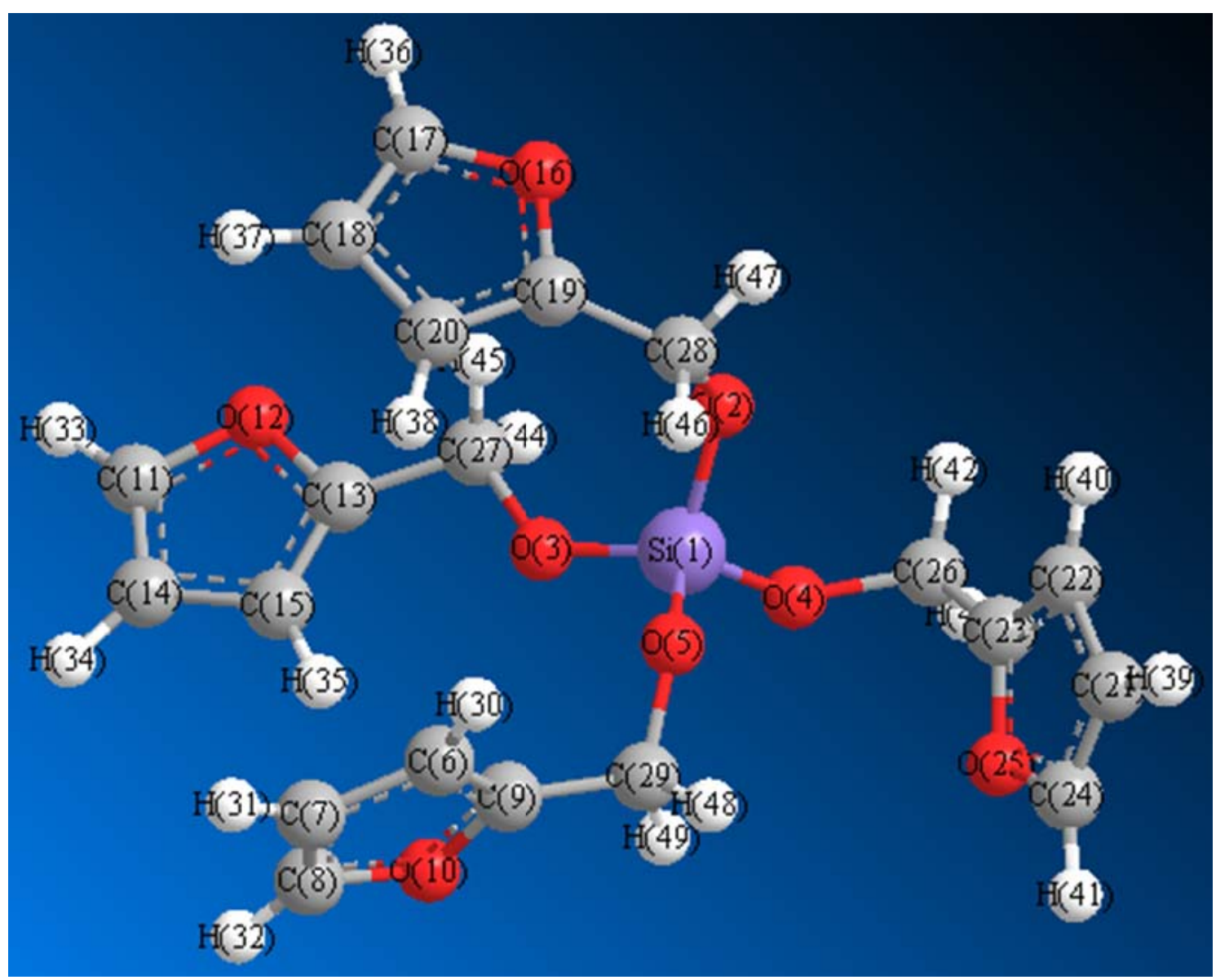

Fig. 4 The structure of the molecule of tetrafurfuryloxysilane $\operatorname{Si}\left(\mathrm{OCH}_{2}\left(\mathrm{C}_{4} \mathrm{H}_{3} \mathrm{O}\right)\right)_{4}$. 
Table 1 Results of minimization the energy characteristics of a molecule of tetrafurfuryloxysilane $\operatorname{Si}\left(\mathrm{OCH}_{2}\left(\mathrm{C}_{4} \mathrm{H}_{3} \mathrm{O}\right)\right)_{4}$.

\begin{tabular}{lllll}
\hline \multirow{2}{*}{ Parameters } & \multicolumn{2}{c}{ The starting basis } & \multicolumn{2}{c}{ Basis after molecular dynamics } \\
\cline { 2 - 5 } & Iteration 599 & Iteration 599+2 & Iteration 189 & Iteration $189+2$ \\
\hline The bonds deformation & 1.0707 & 1.0648 & 1.0720 & 1.0738 \\
The deformation of bond angles & 48.2545 & 48.2542 & 48.7845 & 48.7835 \\
Deformation of tensile-bending & -0.4262 & -0.4203 & -0.3812 & -0.3815 \\
The deformation of the torsion angles & -11.0329 & -11.0331 & -11.1505 & -11.1506 \\
Non van der Waals interactions & -8.8831 & -8.8831 & -15.6637 & -15.6636 \\
van der Waals interactions & 10.4449 & 10.4452 & 10.2485 & 10.2477 \\
The dipole-dipole interaction & 5.3752 & 5.3750 & 4.9159 & 4.9159 \\
The total energy of the molecule, $\mathrm{kJ} / \mathrm{mol}$ & 187.582 & 187.580 & 158.368 & 158.367 \\
\hline
\end{tabular}

To calculate the topology and chemical properties of tetrafurfuryloxysilane molecules $\mathrm{Si}\left(\mathrm{OCH}_{2}\left(\mathrm{C}_{4} \mathrm{H}_{3} \mathrm{O}\right)\right)_{4}$ used different computational methods. Computational chemistry covers a variety of mathematical techniques which are divided into two broad categories:

- Molecular mechanics-apply the laws of classical physics to the atoms in the molecule without explicit consideration of the electrons. For these calculations we used the method MM2 and MMFF94.

- Quantum mechanics is based on the Schrödinger equation for the description of a molecule with an explicit treatment of the electronic structure. In turn the quantum mechanical methods can be divided into two classes: $A b$ initio (nonempirical) and semi-empirical. In the Ab initio method was used the process GAMESS Interface-The General Atomic and Molecular.

Electronic Structure System. In the case of semi-empirical methods we used, a detailed Huckel method (Huckel).

The calculation of atomic charges, which was obtained from electrostatic potentials, provides useful information about the chemical activity. The atomic point charges, give a better idea of the probable locations of attack by chemical interaction of molecules with external agents.

These results indicate heterogeneity furfuryloxy functional groups, which in its turn indicate the possibility of stepwise mechanism, of molecular interaction tetrafurfuryloxysilane between themselves and with the surrounding water molecules during its hydrolysis. Furthermore, the molecule of tetrafurfurylhydroxy-silane is not symmetrical, which also leads to the possibility of stepwise mechanism of nanophase's formation and nanostructuring of polysilicate composite coatings in its preparation.

It was obtained optimal composition of the polysilicate composite coatings, which has increased strength, durability, density and crack resistance. We investigated the diffusion permeability of material and its chemical resistance in various aggressive environments.

\section{Nanostructured Polysilicate Composite Coating}

It is known that the polysilicate composite coating consists of a binder, hardener and polymer additive, fine and coarse filler. Water soluble sodium or potassium silicate glass (with density of $1.38 \div 1.4$ $\mathrm{g} / \mathrm{cm}^{3}$ ) used as the basis for the binder. Technical sodium fluorosilicate is used in most cases as a hardener. The fillers are natural or artificial materials having a high acid resistance, particularly quartz, zircon, diabase, basalt, granite, andesite etc. [14-16].

Polysilicate composite coating has a number of important operational characteristics: high density, fire resistance, resistance to acids, so it is widely used as a facing material for chemical equipment and installations.

However, serious disadvantages of these coatings are their low strength and high shrinkage. By introduction of special organic additives silicates, such as TPS, we can achieve a significant increase in the 
density and strength of the silica matrix in aggressive environments by strengthening contacts between the structural elements of the composition [17].

Optimal composition of coating was obtained. It has a high strength, durability, density and fracture toughness. We investigated the diffusion properties of the coating and chemical resistance in a variety of aggressive environments.

The optimal composition of polysilicate composite coating was determined. The criterion for the optimal composition of composite coating was the conditions of the lowest flow of binder, good machinability, high density and strength. Optimization of the process is generally preceded by a determination of the optimal amount of the silicate binder and addition of monomer. The results of the experiments are shown in Table 2.

From the presented data it is seen that even a small change in the amount of silicate binder greatly changes technical characteristics of the composite coating. Reducing the amount of binder in the mixture reduces the mobility of 2.5 times and about 5 times, increasing its stiffness. Influence of TPS monomeric addition is examined for plastic mixture containing $1 \%$ binder (Table 3 ).

The results of the study clearly demonstrate that the introduction of additive increases the stiffness of mixture.

Influence of the content of silica binder on strength of composite coatings was determined for a composition with the monomer additive and without it. In the first case, as a starting sample was used plastic mixture having the minimum content of the binder $(10$ $\div 13 \%$ ) and the TFS additive in an amount of $3 \%$ by weight of the binder. The results of the experiments are shown in Fig. 5.

Experiments have shown that the strength of the samples of the coating material increases with decreasing content liquid glass binder in the whole measurement range. Reduction of the binder amount only $3 \%$, leads to an increase of the coating material strength by approximately $25 \%$. It can be assumed that this phenomenon is associated with the film thickness of the binder, which coats the grains of the filler. Thus, with decreasing film thickness, the adhesive strength of the interaction is increased, thus increasing the strength and density of the mixture.

It should be noted that the introduction of TPS additives in an amount of $0.3 \%$ in the composite coating increases the strength and density of the material by approximately $50 \%$ over the entire range studied binder content [18, 19].

A lifetime polysilicate nanocomposite coating in aggressive environments depends on the diffusion rate of reactive reagents, which passes through the imperfections of the molecular structure of the binder, due to the impact of temperature and environmental pressure. So determination of the diffusion coefficient in the composite coating, a certain period of time will

Table 2 Influence of binder content on the mobility and stiffness of the mixture during manufacturing of polysilicate composite coating.

\begin{tabular}{llll}
\hline Content of silica binder, $\%$ & Mobility of the mixture, $\mathrm{cm}$ & Stiffness of mixture, $\mathrm{s}$ & Characteristics of mixture \\
\hline 13 & 15 & 15 & The good workability \\
12 & 12 & 10 & Plastic mixture \\
\hline 11 & 6 & 23 & Dry mix \\
\hline
\end{tabular}

Table 3 Influence of TFS additive on mobility and stiffness of mixture.

\begin{tabular}{lll}
\hline TPS mass fraction, $\%$, in the liquid glass & Mobility of SPC mixture, $\mathrm{cm}$ & Stiffness of SPC mixture, $\mathrm{s}$ \\
\hline No additive & 6 & 23 \\
2 & 4 & 24 \\
3 & 2 & 28 \\
6 & 0 & 32 \\
\hline
\end{tabular}




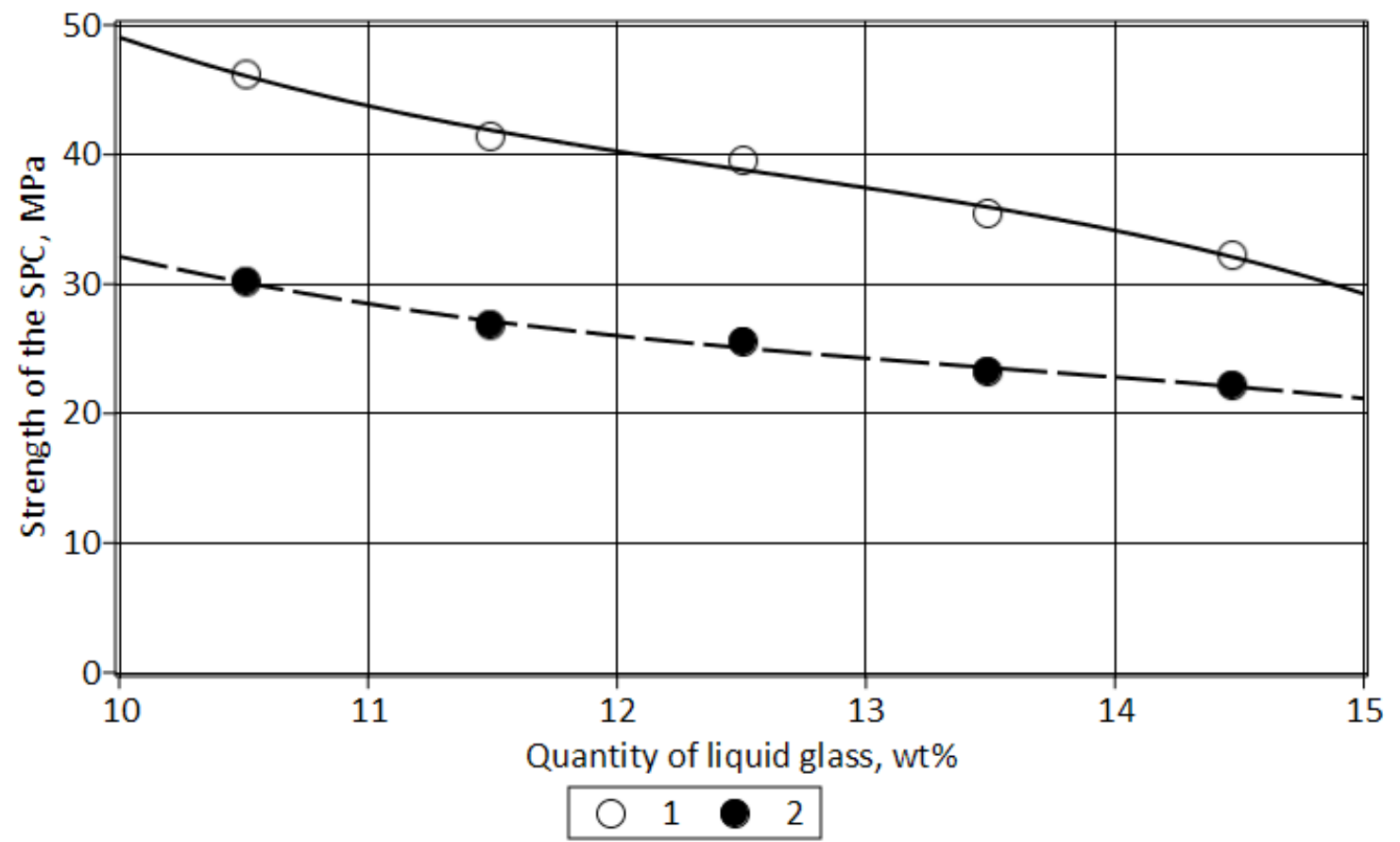

Fig. 5 Changing the strength poly silicate composite coating according to the quantity of liquid glass: 1—Material with the addition of TFS; 2 -Material without the additive.

be important to assess the effect of additives monomers, and setting the maximum allowable concentration of substances causing corrosion.

We investigated the diffuse penetration into the composite coating in a neutral aqueous medium, which is the most aggressive for compositions based on liquid glasses. The results of testing samples of polysilicate nanocomposite coatings for various compositions are shown in Fig. 6.

Obviously, the diffusion penetration of modified TFS additive into the material is considerably lower than in a composition containing additive of FA. Thus the process rates for these samples also vary considerably, especially in the initial period. The diffusion coefficients obtained by the method of sorption, were calculated for two periods of exposure in water, 7 and 30 days (Table 4). Thus, we can conclude that the introduction of furan series modifiers, in a silicate coating composition, reduces the rate of diffusional penetration of aggressive media.

There were used optimum compositions including monomeric additives FA and TFS (see Table 4) to study the effect of weakly acidic environment on the properties of polysilicate nanocomposite coatings. Medium concentration corresponded aqueous solutions of sulfuric and hydrochloric acids used for etching metals. Corrosion resistance of materials assessed by change of the compressive strength of material samples after $3 \div 18$ months exposure in environments with an interval of 3 months. The effects of the monomer type additives strength of poly-silicate nanocomposite coatings after 18-month exposure samples of materials in a weakly acidic aqueous medium are shown in Table 5.

The presented data show that small change in the content of liquid glass dramatically changes the technological characteristics of poly-silicate nanocomposite coatings. With the decrease in the amount of binder, the strength and density of the poly-silicate nanocomposite coatings increase. The optimum composition of the material includes an $11.23 \%$ of water glass and $0.34 \%$ of monomer addition (FS or TFS). Poly-silicate nanocomposite coatings composition modified with TFS additive has high compressive strength and high deformability. Introduction of the monomer addition TFS into the 


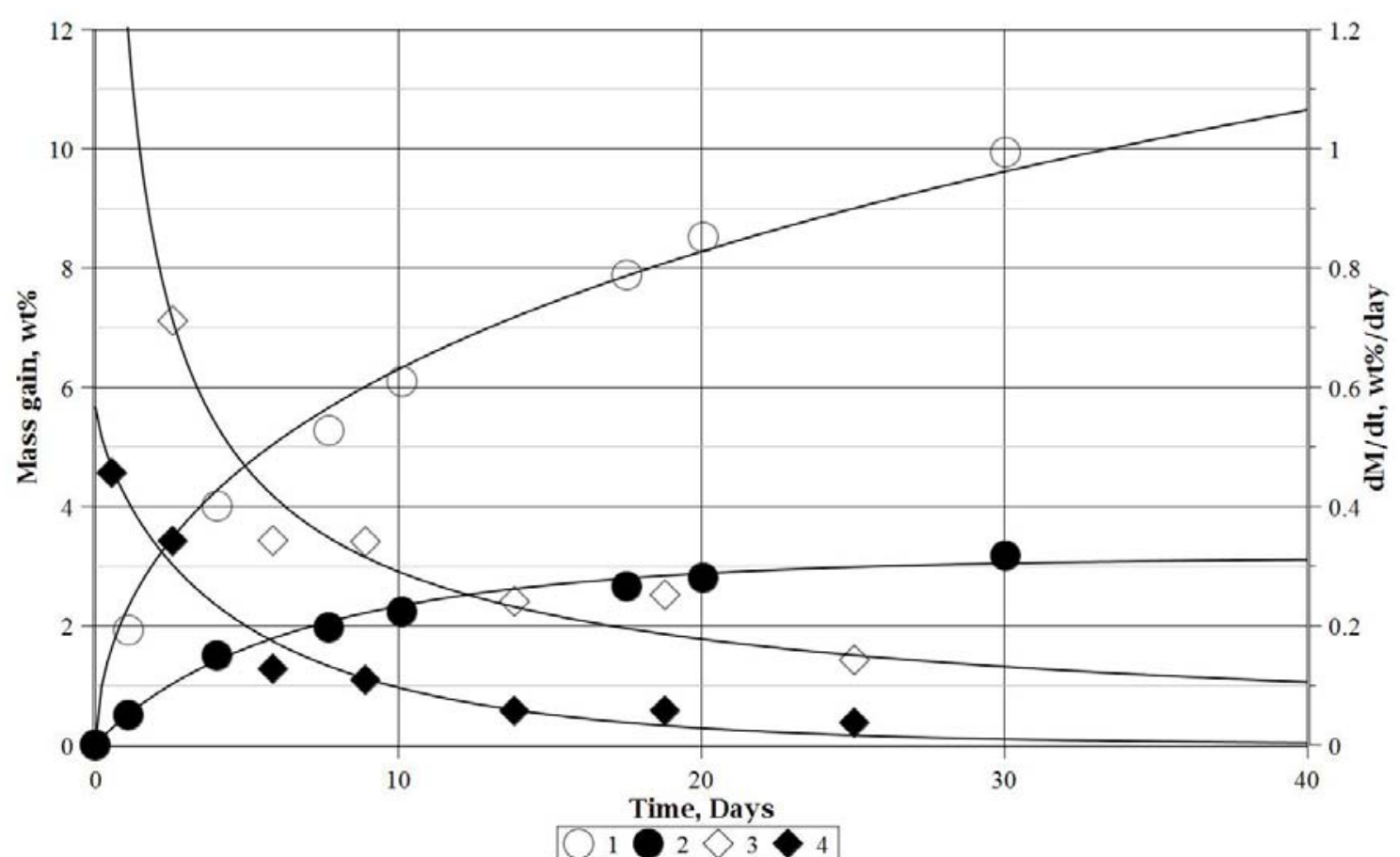

Fig. 6 Mass change $(1,2)$ and rate of mass change $(3,4)$ in water for samples of polysilicate nanocomposite coatings: 1,3-Sample modified FA; 2,4-Sample modified TFS.

Table 4 The diffusion coefficients of the samples of poly-silicate nanocomposite coatings in aqueous medium.

\begin{tabular}{lll}
\hline \multirow{2}{*}{ Material type } & \multicolumn{2}{c}{ The diffusion coefficients, $10^{-7} \mathrm{~cm}^{2} / \mathrm{s}$} \\
\cline { 2 - 3 } & After 7 days & After 30 days \\
\hline Sample modified FA & 8.77 & 8.91 \\
Sample modified TFS & 0.74 & 0.25 \\
\hline
\end{tabular}

Table 5 Compressive strength of samples poly-silicate nanocomposite coatings (MPa) after exposure to the corrosive environment.

\begin{tabular}{llll}
\hline \multirow{2}{*}{ Type of additives } & \multicolumn{3}{c}{ Aggressive environment } \\
\cline { 2 - 4 } & - & $2 \% \mathrm{H}_{2} \mathrm{SO}_{4}$ & $2 \% \mathrm{HCl}$ \\
\hline TFS & 33.8 & 36.3 & 34.6 \\
FA & 25.3 & 25.0 & 24.8 \\
\hline
\end{tabular}

material increases the stiffness of the mixture and very significantly decrease the shrinkage deformations. Additives of furan series reduce the diffusion penetration of aggressive environment into the structure of poly-silicate nanocomposite coatings and improve its corrosion resistance.

\section{Conclusion}

Obtained optimum composition of protective coatings based on organic water-soluble silicates having high strength, durability, density and fracture toughness. Diffusion permeability of these coatings and chemical resistance in a variety of aggressive environments were investigated.

Introduction into the composite coating $0.3 \%$ additive of tetrafurfuryloxysilane increases the strength and density of the material approximately $50 \%$ throughout the range of investigated content of organic water-soluble silicates. Plastic composite mixture allows making products of any geometry. It should be noted that the compressive strength and deformability of samples of the composite coatings modified TFS additive were maximal. Studies had shown that the introduction of monomeric additives 
leads to drastic reduction of shrinkage strain. Shrinkage of the composite coating at the age of 28 days was only $0.06 \%$, while the content in the mixture of $3 \%$ TFS.

The formation of composite coating structure is accompanied by intense compression of the binder gel by capillary forces fluid intermicellar. To the mixture, without the presence of monomer addition, such liquid is water. Compression of the gel leads to a maximal shrinkage deformation from the start of hardening mixture. Additives introduction of FA or TFS in the reaction mixture leads to substantial reduction of the influence of capillary forces by reducing the surface tension of the liquid in the capillaries.

A small change in the content of organic water-soluble silicates dramatically changes the technological characteristics of composite coating. With the reduction of the content of organic water-soluble silicates the strength and density of composite coating increase. The best composition of composite coating includes: $11.23 \%$ silicate binder and $0.34 \%$ of monomeric additives (FA or TFS). The composition of the composite coating modified by the addition of TFS has a high compressive strength and high deformability. Introduction into the composite coating composition the monomeric TFS additives lead to increased stiffness of the mixture and to very significant reduction of shrinkage strain. Additives of furan series compounds are effective in reducing of the diffusion penetration of aggressive environment in the composite coating and increase its resistance to corrosion.

\section{Acknowledgment}

The authors express their gratitude to Mr. Omer Yagel, VP Business Development and Training DigiSec Ltd., official representative of the company Maplesoft, Department of Waterloo Maple Inc. in Israel, for the opportunity use the software product of this company, program of analytical computations-Maple.

\section{References}

[1] Kudryavtsev, P., and Figovsky, O. 2014. "Advanced Nanomaterials Based on Soluble Silicates." Journal Scientific Israel-Technological Advantages 16 (3): 38-76.

[2] Korneev, V. I., and Danilov, V. V. 1996. Soluble and Liquid Glass. St. Petersburg: Stroyizdat.

[3] Maltsev, G. N. 2000. Corrosion and Protection of Equipment against Corrosion. Penza: Penza State University.

[4] Figovsky, O., Borisov, Yu., and Beilin, D. 2012. "Nanostructured Binder for Acid-Resisting Building Materials." J. Scientific Israel-Technological Advantages 14 (1): 7-12.

[5] Figovsky, O., Beilin, D., and Ponomarev, A. 2012. "Success of Nanotechnology Applications in Building Materials." Nanotechnology in Construction 3: 6-21.

[6] Kudryavtsev, P., and Figovsky, O. 2014. Nanomaterials Based on Soluble Silicates. Monograph: LAP Lambert Academic Publishing. ISBN 978-3-659-63556-4.

[7] Figovsky, O., and Kudryavtsev, P. 2014. "Advanced Nanomaterials Based on Soluble Silicates." J. Scientific Israel-Technological Advantages 16 (3): 38-76.

[8] Beylin, D. A. Borisov, Yu. M., Figovsky, O. L., and Surovtsev, I. S. 2009. Nano structuring binder composite building materials. Patent RF 2408552.

[9] Novak, M. B., and Ellsworth, M. W. 1993. Composite materials of interpenetrating inorganic and organic polymer networks. US patent 5,254,638.

[10] OnozaWa, S.-Y., Fukaya, N., Saitou, K., Sakakura, T., Yasuda, H., Takagi, Y., and Ueda, M. 2013. Organic Inorganic Composite Material and Utilization Thereof. US patent 8,507,630.

[11] Iler, R. K. 1979. The Chemistry of Silica. New York: John Wiley and Sons.

[12] Figovsky, O., and Beilin, D. 2013. Advanced Polymer Concretes and Compounds. Tailor \& Francis Group: CRC Press.

[13] Holtje, H.-D., Sippl, W., Rognan, D., and Folkers, G. 2008. Molecular Modeling. Basic Principles and Applications. WILEY-VCH Verlag GmbH \& Co. KGaA.

[14] Figovsky, O., Beilin, D., and Ponomarev, A. 2012. "Success of Nanotechnology Applications in Building Materials." Nanotechnology in Construction 3: 6-21.

[15] Figovsky, O., and Olkhovik, E. 2001. "Method of Predicting Concrete Creep." Journal of the Mechanical Behavior of Materials 12 (2).

[16] Figovsky, O. L. 1994. "Advanced Composite Materials Based on Silicate-Polymers Matrix." Proceedings of Pan-Asian Aerospace Technology Conference, Beijing, China. 
[17] Kudryavtsev, P., Figovsky, O., and Kudryavtsev, N. 2016. "Advance in Nanocomposites Based on Hybrid Organo-Silicate Matrix." Chemistry \& Chemical Technology 10 (1): 45-53.

[18] Solomatov, V. I., Bobryshev, A. N., and Khimmler, N. G. 1988. Polymer Composite Materials in Construction.
Moscow: Stroyizdat.

[19] Figovsky, O., and Beilin, D. 2009. "Improvement of Strength and Chemical Resistance of Silicate Polymer Concrete." International Journal of Concrete Structures and Materials 3 (2): 97-101. DOI: 10.4334/IJCSM.2009.3.2.097. 